\title{
Interstitial pneumonia with autoimmune features (IPAF) and radiological findings suggestive of lymphocytic interstitial pneumonia (LIP) - case report
}

The authors declare no financial disclosure

\begin{abstract}
Interstitial pneumonia with autoimmune features (IPAF) is a term to describe individuals with both interstitial lung disease (ILD) and combinations of other clinical, serologic, and/or pulmonary morphologic features, which presumably originate from an underlying systemic autoimmune condition, but do not meet current rheumatologic criteria for a defined connective tissue disease (CTD). Predominantly, interstitial pneumonia arises in the course of an established CTD, but it is not so rare for the ILD to be the first, and possibly the one and only manifestation of a latent CTD. Lymphocytic Interstitial Pneumonia (LIP) is an uncommon disease, characterized by infiltration of the interstitium and alveolar spaces of the lung by lymphocytes, plasma cells and other lymphoreticular elements. The cause of LIP is still unknown but it could be also a manifestation of CTD. Clinically, it is highly variable, from spontaneous resolution to progressive respiratory failure and death despite glucocorticoid treatment. Since there are no recent standards for the management of LIP, the disease is treated empirically.

We report a case of a HIV-negative 54-year-old woman, who was suspected of LIP according to clinical features and radiological findings. Positive laboratory results were highly suggestive of underlying autoimmune process, but did not fulfil the criteria of any particular CTD. Because of severe general condition of the patient, immunosuppressive treatment was started immediately, without further invasive diagnostics including lung biopsy, which is required for a definitive diagnosis. We present two-year observation of the patient with all our doubts concerning clinical proceedings.
\end{abstract}

Key words: Iymphocytic interstitial pneumonia, immunosuppressive treatment, clinical suspicion, interstitial pneumonia with autoimmune features

Adv Respir Med 2017; 85: 46-50

\section{Introduction}

Interstitial pneumonia with autoimmune features (IPAF) is a term given to characterize a heterogeneous group of patients with idiopathic interstitial pneumonia (IIP) who have a clinical flavour of underlying connective tissue disease (CTD) but do not meet the current American College of Rheumatology criteria for it. The features from three domains are crucial for establishing diagnosis of IPAF: a clinical domain consisting of specific extra-thoracic features, a serologic domain including specific autoantibodies, and a morphologic domain comprising specific chest imaging, histopathologic or pulmonary physiologic features. Interstitial pneumonia is one of the most common and known clinical manifestations of CTD. It can take different forms, including lymphocytic interstitial pneumonia (LIP). LIP is an uncommon disease that is histopathologically characterized by infiltration of the interstitium and alveolar spaces of the lung by lymphocytes,

\footnotetext{
Address for correspondence: Alicja Płóciniczak, Department of Pulmonolgy, Allergology and Respiratory Oncology, Poznan University of Medical Sciences, Poland. Hospital of Lord's Transfiguration, Szamarzewskiego 82,60-569 Poznań, e-mail: petit_rayon@tlen.pl

DOI: 10.5603/ARM.2017.0009

Received: 14.12 .2016

Copyright (C) 2017 PTChP

ISSN 2451-4934
} 
plasma cells, and histiocytes [1]. LIP's association with several autoimmune disorders including Sjögrens syndrome, systemic lupus erythematous (SLE), rheumatoid arthritis [2] and pernicious anaemia is well known. In general, changes characteristic of LIP have been reported in the course of viral infection, especially caused by HIV and EBV [3]. However, some of the cases remain idiopathic, when all systemic diseases are excluded and classified as IIP (idiopathic interstitial pneumonia) [4]. Data concerning mortality and morbidity of the LIP are underestimated and inaccurate due to the lack of reported follow-up and rarity of the disease. Because of non-specific symptoms (non-productive cough, dyspnoea, crackles) and radiological changes (ground-glass appearance, bibasilar interstitial marking, honeycombing, development of fibrosis, bronchiectasis, bullae or cystic changes), open lung biopsy should be considered to verify the diagnosis and/or exclude other possible causes [3]. Differential diagnosis includes: pseudolymphoma, lymphomatoid granulomatosis, follicular bronchiolitis, primary pulmonary lymphoma, bronchocentric granulomatosis, IgG4-related disease, hypersensitivity pneumonitis (e.g, characterized by the non-specific interstitial pneumonia [NSIP] pattern) and pneumonia caused by Pneumocystis jirovecii, cytomegalovirus and mycobacteria in HIV-infected patients [5].

Due to the rarity of the disease and variety of possible aetiology, data regarding LIP treatment commonly come from case series' reports and therefore are still uncompleted. No treating standards have been established.

We present a case of a 54-year-old patient suspected of LIP, most likely secondary to connective tissue disease (IPAF). The suspicion was based on clinical presentation, radiological findings and some laboratory abnormalities.

\section{Case report}

A fifty-four-year-old woman was admitted in September 2013 to the Pulmonology Department due to increasing rest dyspnoea and dry cough. First symptoms of respiratory distress on exertion occurred in January 2013. At that time the patient was diagnosed with interstitial pulmonary changes of unknown aetiology and secondary respiratory failure. Afterwards she has been given several courses of antibiotics due to the exacerbation in the course of the disease of infection origin. She was a former smoker (30 pack-years). In her past medical history she reported pain in both hip-joints, which started in 1996. At first it was treated with NSAIDs by the GP with no rheumatological consultation. In 2000 hip replacement was performed, no symptoms afterwards. Furthermore, 11 years ago she had thyreoidectomy due to goitrous thyroiditis. Neither other significant medical history, nor exposure to irritants, was reported. Her family and occupational history were unremarkable. When referred to the Pulmonology Department, she was in severe general condition, afebrile, with tachypnoe $30 / \mathrm{min}$, heart beat $91 / \mathrm{min}$, blood pressure $90 / 60 \mathrm{~mm} \mathrm{Hg}$ and saturation $71.6 \%$. On the examination, central cyanosis was observed, when sitting, she tended to lean forward, she had clubbed fingers, dry tongue. No skin lesions were found. There were crackles in both lung fields on auscultation, heart rhythm was normal with no pathological sounds. No lymphoadenopathy was observed. The abdomen and extremities were unremarkable.

The results of laboratory studies were as follows: complete cell count, liver function, renal function, brain natriuretic peptide, TSH, the blood urea nitrogen, electrolyte test results and urine analysis were all within normal levels. The arterial blood gas revealed respiratory failure type 1: $\mathrm{pH} 7,503 ; \mathrm{pCO}_{2}: 36.4 \mathrm{~mm} \mathrm{Hg} ; \mathrm{pO}_{2}: 34.1 \mathrm{~mm}$ $\mathrm{Hg} ; \mathrm{HCO}_{2}: 27.4 \mathrm{mmol} / \mathrm{L}$; sat: $71.6 \%$. Coagulation results showed elevated level of D-dimers up to $1932 \mathrm{ng} / \mathrm{mL}$. C-reactive protein was elevated to $42.50 \mathrm{mg} / \mathrm{L}$. The total protein was normal $(74.5$ $\mathrm{g} / \mathrm{L}$ ), whereas the albumin level decreased to $26.6 \mathrm{~g} / \mathrm{L}$ and gamma-globulin increased to 25.0 g/L. HIV antibody was negative. The rheumatoid factor was positive, but the results of anti-CCP antibodies were negative. The antinuclear antibody was over 1:10,000 with no anti-SSA, anti-SSB, anti-nRNP, anti-Sm, anti-Jo1 antibodies. However, anti-PM-Scl, anti-dsDNA, anti-nucleosoms, anti-histons antibodies were found. No laboratory tests concerning lupus anticoagulant, anticardiolipin antibodies nor LE cells were performed.

The body plethysmography showed a low total lung capacity [TLC 65.9\%]. The patient was not able to perform other pulmonary function tests (spirometry, diffusing capacity for carbon monoxide) due to severe rest dyspnoea.

On the echocardiography examination, left ventricle relaxation was decreased and pulmonary hypertension with right ventricular systolic pressure (RVSP) of $60 \mathrm{~mm} \mathrm{Hg}$ was found. High-resolution CT showed massive interstitial changes in both lung areas, diffuse ground-glass opacities, cysts up to $3 \mathrm{~cm}$ mostly located in the lower lobes 

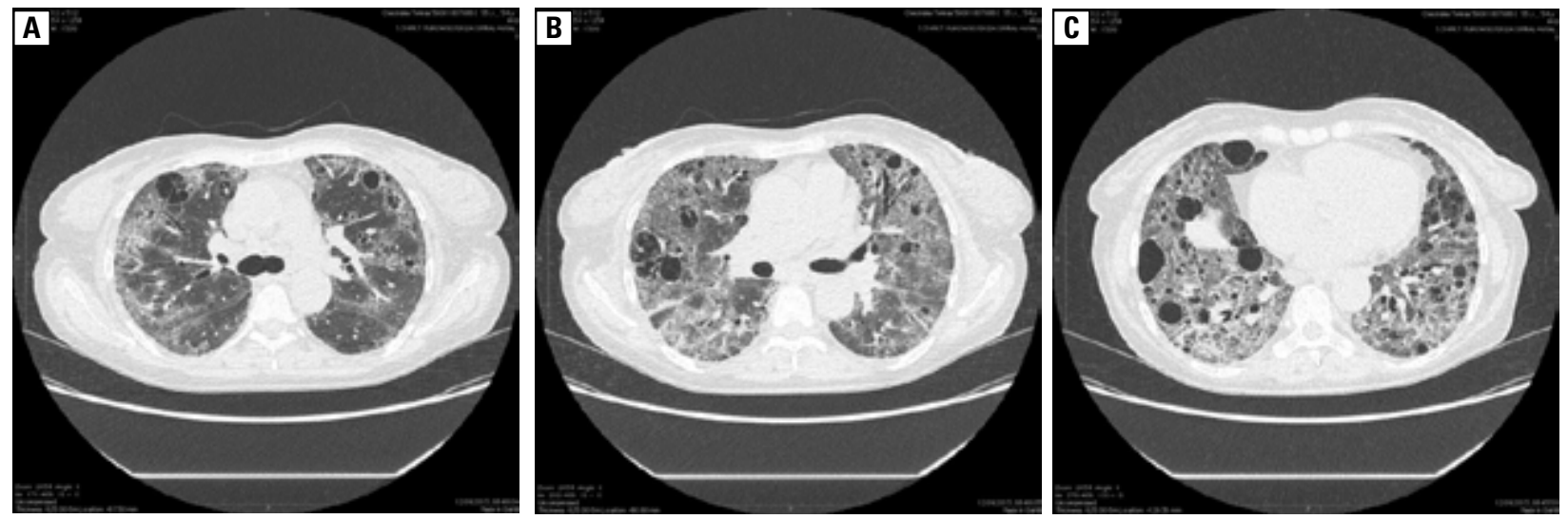

Figure 1. Radiological changes seen in HRCT in September 2013 (thanks to the Radiology Ward of SKPP UM in Poznań)
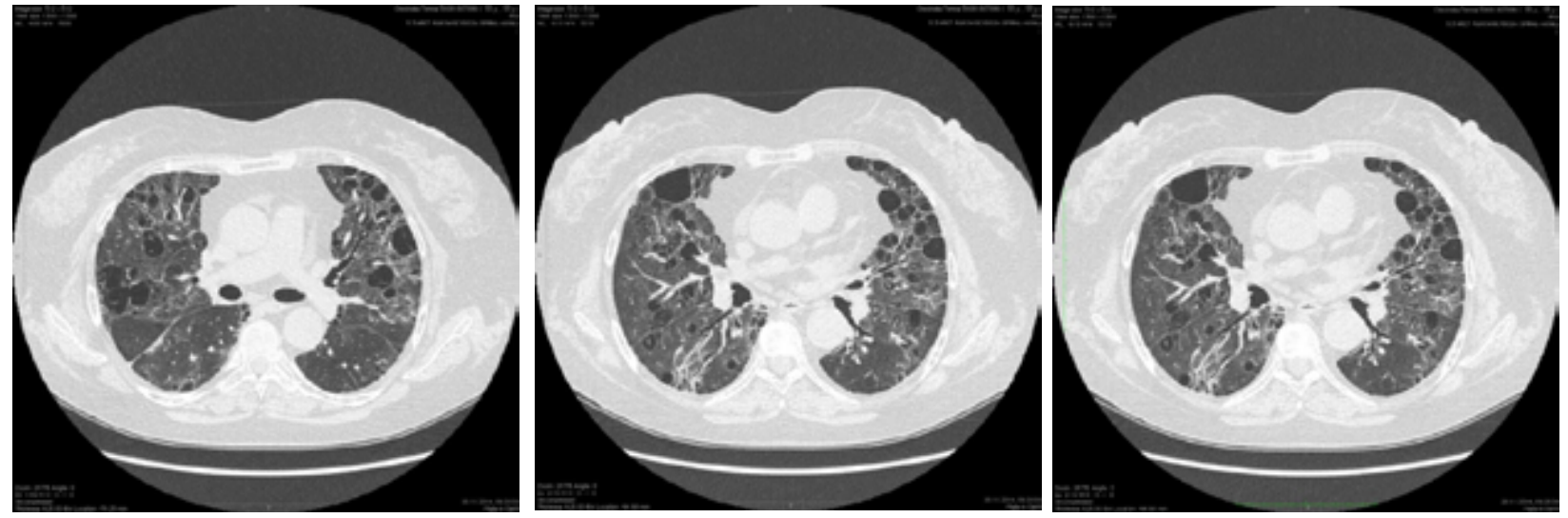

Figure 2. Radiological changes seen in HRCT in the follow-up in July 2014 (thanks to the Radiology Ward of SKPP UM in Poznań)

of both lungs. All these radiological findings are very suggestive of LIP (Fig. 1).

According to rheumatological consultation, capillaroscopy and Schirmer's test were performed and no abnormalities were observed.

Because of severe general condition of the patient, bad results of the pulmonary function tests, coexisting respiratory failure and pulmonary hypertension, she was disqualified from further diagnostics including bronchoscopy with BAL and invasive procedures (VAT, transbronchial lung biopsy). The patient was diagnosed with the suspicion of LIP based on symptoms and typical radiological changes in the lung fields. Even though the connective tissue origin of the LIP in the described patient was highly suspected, in rheumatological opinion, the results presented above were insufficient to diagnose any particular disease. The anti-inflammatory treatment was started in hospital using systemic corticosteroids (500 mg of metyloprednisolone in pulses for 3 days) and continued initially with $40 \mathrm{mg}$ of prednisone a day with gradual reduction of the dose at home. Because of coexisting chronic respiratory failure, the patient was advised to undergo the oxygen therapy ( $1.5 \mathrm{l} / \mathrm{min}$ as needed).

After 10 months of taking systemic glucocorticosteroids (GCs) with the minimal dose of 25 mg prednisone, the patient reported pathological spine fracture. Then, she was admitted again to the Pulmonology Department for the assessment of the course of disease. On the follow-up examination, significant improvement with reduction of interstitial changes on the chest CT (Fig. 2) and RVSP in echocardiography (to $33 \mathrm{~mm} \mathrm{Hg}$ ) was observed. What is more - the patient's clinical status was good enough to perform all pulmonary tests including spirometry, body plethysmography and DLCO measurements. Forced vital capacity (FVC) and TLC results were within normal range, but DLCO suggested severe diffusion disorder $(21.6 \%$ of predictive value). Apart from advantages, the immunosuppressive treatment caused some side effects such as the aforementioned spine fracture but also problems with vision, significant abdominal obesity and vessels fragility. All of them 
markedly reduced the patient's quality of life. Then, the therapy with azathioprine was initiated, so as to decrease the dosage of GCs. Ultimately, the dose of methyloprednisolone was reduced to $4 \mathrm{mg}$ daily and administration of azathioprine $100 \mathrm{mg}$ a day was continued.

\section{Discussion}

In 2015, ERS/ATS published a statement in which the necessity of introducing concept of IPAF was underlined. IPAF is recommended for all clinical situations, when the interstitial changes in the lungs are followed by autoantibodies' positive tests with no particular CTD. However, there are still many doubts concerning the matter, as the classification is available for a very short period of time. Several months ago another article referring to IPAF was published revealing queries about the terminology. The authors agreed with Fischer et al. [2] that the term 'IPAF' is necessary, but emphasized that the terminology is still not validated and there is still a huge group of patients that do not meet all criteria for CTD or IPAF [3]. To fulfil the criteria for IPAF, the patient must have radiological features and/or lung biopsy. If the patient fails to meet criteria for a specific CTD, and IIP are excluded, he must have at least one characteristic from at least two out of: clinical, serological and morphological domains.

In general, due to ATS/ERS classification, LIP develops mainly secondarily to viral infection [6], it may be of connective tissue disease origin, but several studies still suggest there may be cases of idiopathic LIP $[4,6]$. This fact has been confirmed in the last International Multidisciplinary Classification of the Idiopathic Interstitial Pneumonias (IIPs), where-idiopathic LIP was included in the category of rare IIPs [7]. In the presented patient, HIV infection was excluded at the beginning, there were no symptoms suggestive of EBV infection or immunodeficiency. However, her interstitial lung disease was associated with autoantibody production. The patient did not meet the criteria of a particular connective tissue disease, but HRCT radiological pattern suggestive of LIP and serological features fulfilled the criteria of IPAF [2].

According to the 2002 ATS/ERS classification of IIPs, diagnosis of LIP was possible when clinical, imaging and histopathologic criteria occurred, so thoracoscopic or open-lung biopsy specimens are required [8]. The question is whether the radiographic pattern and symptoms in HIV-positive children are sufficient to diagnose
LIP without invasive procedures [9], and how to classify adult patients that meet most of the criteria apart from pathological findings? How to diagnose patients who are disqualified from further invasive procedures because of severe clinical condition? Similar dilemma was highlighted in the newest classification of IIPs of 2013 in which the historical gold standard of histological diagnosis was replaced by a "dynamic integrated approach" using multidisciplinary discussion. The multidisciplinary approach does not lessen the importance of lung biopsy in the diagnosis of IIPs. It defines the settings where biopsy is more informative than HRCT and those where biopsy is not needed [7]. In this document, LIP is not listed as an example when invasive diagnosis is not required, but it did not give the answer to doubts what to do in the case of suspicion of disease and disqualification from invasive procedures. Regarding the above, the typical clinical presentation and CT images without the results of the biopsy were sufficient just to suspect LIP in the described patient without establishing a final diagnosis.

Due to the rarity of the disease, the data concerning morbidity, mortality and treatment are uncompleted. Controlled clinical trials have not been reported for LIP. Data related to therapeutic options are available through case reports. The treatment of adults with LIP differs depending on the severity of the patient's symptoms, presented impairments in pulmonary function tests, and the presence or absence of a coexistent disease. So far no specific guidelines have been established. In general, most data originate from patients with autoimmune disease in whom systemic glucocorticosteroids have been administered. The optimal dose and duration of such therapy are also uncertain. The primary objective is to obtain the maximum effect with the minimum dose.

It is suggested that the initial dose of prednisone 0.75 should amount to $1 \mathrm{mg} / \mathrm{kg}$ given once daily (max. $100 \mathrm{mg}$ ). The dose is continued for 8 to 12 weeks, then slowly reduced to the minimal one. The therapeutic effects are controlled by patient's symptoms, radiological findings on HRCT and pulmonary function tests (FVC, TLC, DLCO). As systemic steroid therapy does not meet expected effects in all patients, the next step is to enclose immune suppressants like azathioprine or cyclosporine A. That seems to be the most successful therapy $[6,10,11]$, however, there is a report that has described good response to cyclosporine A after no improvement with steroids [11]. Furthermore, impro- 
vement in LIP associated with common variable immunodeficiency syndrome (CVID) treated with immunoglobulin without steroids and immune suppressants has also been published [12]. In 2013, a good response to Mycophenolate Mofetil was reported among patients with idiopathic LIP alone, as well as both LIP and connective tissue disorder [13, 14]. In Poland, Mycophenolate Mofetil is not registered for the treatment of LIP. In this case, no particular connective tissue disease has been diagnosed, probably due to permanent steroid therapy. Moreover, chronic treatment with oral steroids $(25$ mg of prednisone) has caused many side effects such as osteoporosis, obesity, vessels fragility, cataract. On the other hand, lower doses of oral steroids have increased symptoms and caused deterioration of general condition. Thus, our patient has been given azathioprine, in order to minimize the dose of systemic steroids. In 24-month-long follow-up she seems to improve and her respiratory symptoms are stable.

\section{Conclusion}

In general, LIP is still underdiagnosed and underestimated due to its rarity. Many data concerning pathogenesis, clinical, radiological features and treatment are inexact. As there are so many doubts and uncertainty, the question remains if those cases diagnosed previously as idiopathic interstitial pneumonias when tested positively for any autoantibodies, should be treated as IPAF? The Update of the International Multidisciplinary Classification of the Idiopathic Interstitial Pneumonias of 2013 and other recent publications concerning IIP may be helpful for doctors taking care of such group of the patients regarding diagnostic doubts.

What kind of treatment should be applied so as to extend patients' life expectancy and improve their quality of life? Since there is no standard therapy established, every information on the issue is very valuable. Additional clinical trials and studies are needed. Yet, the most important is to weigh the risk of side effects against potential benefits, and ensure the patient the best quality of life-

\section{Conflict of interest}

The authors declare no conflict of interest.

\section{References:}

1. Koss MN, Hochholzer L, Langloss JM, et al. Lymphoid interstitial pneumonia: clinicopathological and immunopathological findings in 18 cases. Pathology. 1987; 19(2): 178-185, doi: 10.3109/00313028709077131, indexed in Pubmed: $\underline{3453998}$.

2. Fischer A, Antoniou KM, Brown KK, et al. "ERS/ATS Task Force on Undifferentiated Forms of CTD-ILD”. An official European Respiratory Society/American Thoracic Society research statement: interstitial pneumonia with autoimmune features. Eur Respir J. 2015; 46(4): 976-987, doi: 10.1183/13993003.001502015, indexed in Pubmed: 26160873.

3. Collins B, Raghu G. Interstitial pneumonia with autoimmune features: the new consensus-based definition for this cohort of patients should be broadened. Eur Respir J. 2016; 47(4): 1293-1295, doi: 10.1183/13993003.02084-2015, indexed in Pubmed: 27037322.

4. American Thoracic Society/European Respiratory Society International Multidisciplinary Consensus Classification of the Idiopathic Interstitial Pneumonias. Am J Respir Crit Care Med. 2002; 165(2): 277-304, doi: 10.1164/ajrccm.165.2.ats01.

5. www.uptodate.com/contests/lymphoid-interstitial pneumonia; 28.07.2016.

6. Swigris JJ, Berry GJ, Raffin TA, et al. Lymphoid interstitial pneumonia: a narrative review. Chest. 2002; 122(6): 2150-2164, doi: 10.1378/chest.122.6.2150, indexed in Pubmed: 12475860.

7. Travis WD, Costabel U, Hansell DM, et al. ATS/ERS Committee on Idiopathic Interstitial Pneumonias. An official American Thoracic Society/European Respiratory Society statement: Update of the international multidisciplinary classification of the idiopathic interstitial pneumonias. Am J Respir Crit Care Med. 2013; 188(6): 733-748, doi: 10.1164/rccm.201308-1483ST, indexed in Pubmed: 24032382.

8. Vath RR, Alexander CB, Fulmer JD. The lymphocytic infiltrative lung diseases. Clin Chest Med. 1982; 3(3): 619-634, indexed in Pubmed: 6751673.

9. Rubinstein A, Morecki R, Goldman H. Pulmonary disease in infants and children. Clin Chest Med. 1988; 9(3): 507-517, doi: 10.1002/9780470692042.ch12, indexed in Pubmed: 3044686.

10. Kim JY, Park SH, Kim SK, et al. Lymphocytic Interstitial Pneumonia in Primary Sjögren's Syndrome: A Case Report. Korean J Intern Med. 2011; 26(1): 108, doi: 10.3904/ kjim.2011.26.1.108.

11. Cha SI, Fessler MB, Cool CD, et al. Lymphoid interstitial pneumonia: clinical features, associations and prognosis. Eur Respir J. 2006; 28(2): 364-369, doi: 10.1183/09031936.06.00076705, indexed in Pubmed: 16571614 .

12. Arish N, Eldor R, Fellig Y, et al. Lymphocytic interstitial pneumonia associated with common variable immunodeficiency resolved with intravenous immunoglobulins. Thorax. 2006; 61(12): 1096-1097, doi: 10.1136/thx.2004.029819, indexed in Pubmed: 17114373.

13. Abdarbashi P, Abrudescu A. Rare case of idiopathic lymphocytic interstitial pneumonia exhibits good response to Mycophenolate Mofetil. Respir Med Case Rep. 2013; 9: 27-29, doi: 10.1016/j.rmcr.2013.04.004, indexed in Pubmed: 26029625.

14. Fischer A, Brown KK, Du Bois RM, et al. Mycophenolate mofetil improves lung function in connective tissue disease -associated interstitial lung disease. J Rheumatol. 2013; 40(5): 640-646, doi: 10.3899/jrheum.121043, indexed in Pubmed: 23457378. 